Guido Melchior

\title{
Skepticism: the hard problem for indirect sensitivity accounts
}

\author{
[FINAL DRAFT]
}

Published in Erkenntnis, DOI: 10.1007/s10670-013-9432-9

\begin{abstract}
Keith DeRose's solution to the skeptical problem is based on his indirect sensitivity account. Sensitivity is not a necessary condition for any kind of knowledge, as direct sensitivity accounts claim, but the insensitivity of our beliefs that the skeptical hypotheses are false explains why we tend to judge that we do not know them. The orthodox objection line against any kind of sensitivity account of knowledge is to present instances of insensitive beliefs that we still judge to constitute knowledge. This objection line offers counterexamples against the claim of direct sensitivity accounts that sensitivity is necessary for any kind of knowledge. These examples raise an easy problem for indirect sensitivity accounts that claim that there is only a tendency to judge that insensitive beliefs do not constitute knowledge, which still applies to our beliefs that the skeptical hypotheses are false. However, a careful analysis reveals that some of our beliefs that the skeptical hypotheses are false are sensitive; nevertheless, we still judge that we do not know them. Therefore, the fact that some of our beliefs that the skeptical hypotheses are false are insensitive cannot explain why we tend to judge that we do not know them. Hence, indirect sensitivity accounts cannot fulfill their purpose of explaining our intuitions about skepticism. This is the hard problem for indirect sensitivity accounts.
\end{abstract}

\section{Keywords}

Skepticism - Sensitivity — Indirect sensitivity accounts — Keith DeRose — Contextualism

\section{$1 \quad$ Introduction}

$\mathrm{S}$ 's belief that $p$ is sensitive if and only if $\mathrm{S}$ would not believe that $p$, if $p$ were not true. To a first approximation Nozick (1981) provides an analysis of knowledge as follows: $\mathrm{S}$ knows that $p$ iff (1) $p$ is true; (2) $\mathrm{S}$ believes that $p$; (3) If $p$ weren't true, $\mathrm{S}$ wouldn't believe that $p$ and (4) If $p$ were true, $\mathrm{S}$ would believe that $p$. Hence, condition (3) specifies that sensitivity is a necessary condition for knowledge, according to Nozick.

Nozick proposes the following solution to the skeptical argument. Our external world beliefs can be sensitive but our beliefs that the skeptical hypothesis is false are not. Suppose that $\mathrm{S}$ truly believes that there is a computer in front of her. In the nearest possible world where there is no computer in front of $\mathrm{S}, \mathrm{S}$ does not believe that there is a computer in front of her. However, in the nearest possible world where $S$ is a brain in vat (BIV) deceived in falsely believing that there is a computer in front of her, $\mathrm{S}$ still believes that she is not a BIV deceived in falsely believing that there is a computer in front of her. Therefore, it is possible that we have external world knowledge without knowing that the skeptical hypothesis is false.

Nozick's sensitivity account has largely fallen out a favor for at least two reasons: First, it implies that the widely accepted principle of knowledge-closure does not hold. According to sensitivity accounts, it is possible 
that $\mathrm{S}$ knows that $p$, knows that $p$ entails $q$, but still does not know that $q .{ }^{1}$ This fact is often regarded as counterevidence against Nozick's sensitivity account. Second, numerous authors, most prominently Vogel (1987), Sosa (1999), Williamson (2000) and Kripke (2011), have presented instances of insensitive beliefs which we still regard as constituting knowledge. ${ }^{2}$ They conclude that sensitivity is not a necessary condition for knowledge as Nozick claims.

DeRose (1995 and 2010) distinguishes between direct and indirect sensitivity accounts. Direct accounts such as Nozick's simply hold that sensitivity is necessary for knowledge. DeRose defends an indirect sensitivity account that is more modest than Nozick's direct account. He argues that the insensitivity of certain beliefs explains why they fail to be knowledge though he does not want to build a sensitivity condition in the very concept of knowledge.

DeRose's indirect account avoids the two criticized problems of Nozick's direct account. First, DeRose proposes a contextualist solution according to which we have external world knowledge and knowledge that the skeptical hypothesis is false in some contexts and we know neither in the other contexts, but knowledge-closure holds in every context. Second, he does not assume that sensitivity is a necessary condition for knowledge in particular. Therefore, the examples for insensitive knowledge presented in literature need not be counterexamples against his indirect sensitivity account and against his treatment of the skeptical problem. However, I will argue that even granting these advantages, DeRose's solution to skeptical problem still faces serious problems.

\section{DeRose's indirect sensitivity account}

Let's have first a closer look at DeRose's indirect sensitivity account and at his solution to the skeptical problem. According to DeRose $(2010,163)$ we have "some at least fairly general-though perhaps not exceptionless-tendency to judge that insensitive beliefs are not knowledge". DeRose calls this principle SCA. Anti-skeptical hypotheses are such representative cases for which this tendency holds. Hence, the insensitivity of our beliefs of anti-skeptical hypotheses explains why we judge that these beliefs fail to constitute knowledge, although sensitivity might not be a necessary condition for knowledge in general. DeRose $(1995,28)$ finds the conjunction abominable, that while $\mathrm{S}$ does not know that she is not a handless BIV, still, $\mathrm{S}$ knows that she has hands. DeRose rejects this conjunction and endorses a contextualist solution to the skeptical problem: In ordinary contexts persons can have knowledge about the external world and knowledge that the skeptical hypotheses are false. But when it is asserted that a person $\mathrm{S}$ knows that $p$ (or that she does not know that $p$ ), the standards for knowledge tend to be raised, if need be, to such a level that S's belief has to be sensitive to count as knowledge. This is DeRose's "Rule of Sensitivity". By asserting that one knows that the skeptical hypotheses are false, one establishes such high standards and, as a result, the assertion turns out to be false. In these contexts a person neither has knowledge about the external world nor knowledge that the skeptical hypothesis is false.

\footnotetext{
${ }^{1}$ For a detailed presentation of various versions of knowledge-closure and its role in the skeptical argument see David and Warfield (2008).

${ }^{2}$ Kripke (2011) argues that Nozick's sensitivity account allows under certain circumstances that a person knows that there is a red barn in front of her without knowing that there is barn in front of her, which is an absurd consequence. However, it is questionable whether Kripke's case against Nozick is still successful if we take the belief forming method into account as Nozick suggests. See Adams and Clarke (2005) and Baumann (2012).
} 
The most popular line of objection against any kind of sensitivity account of knowledge so far is to present instances of insensitive beliefs that we still judge to constitute knowledge. This orthodox objection line offers counter-examples against the claim of direct sensitivity accounts that sensitivity is necessary for any kind of knowledge. DeRose $(2010,164)$ points out that the reasons that have been given to reject any kind of sensitivity account are almost entirely due to such examples of insensitive knowledge. These examples raise an easy problem for DeRose, since he defends an indirect sensitivity account, which is the claim that there is only a tendency to judge that insensitive beliefs do not constitute knowledge. So DeRose can acknowledge the facts regarding insensitive knowledge presented by orthodox objections but still maintain that these are only exceptions to the tendency which still applies to our beliefs that we are not BIVs. Moreover, even if DeRose had to confess that there is no general tendency to judge that insensitive beliefs are not knowledge at all, he could carry on to claim that in the particular case of our beliefs that we are not BIVs, this tendency still applies and still does the job of explaining our intuition that we fail to know.

I agree with DeRose that providing examples of insensitive knowledge outside the skeptical context might drive him to weaken his point that there is a general tendency to judge that insensitive beliefs are not knowledge, but that he is not forced to abandon his view that insensitivity does the explanatory job in the particular case of skepticism. However, I will argue that DeRose's position regarding the skeptical problem is still not tenable and cannot provide an explanation of our intuitions about skepticism. Orthodox objections against DeRose point out that his sensitivity account does not apply to many cases outside the skeptical context. But the crucial point is that his account cannot fulfill its purpose in the particular context of skepticism. This is the hard problem for indirect sensitivity accounts.

\section{The hard problem for indirect sensitivity accounts}

DeRose compares one's belief that one is not a BIV to one's belief that the animal in the cage in front of one is not a painted mule, or that a newspaper does not mistakenly print that the Cubs won their game last night. In these analogous cases principle SCA (our general but not exceptionless tendency to judge that insensitive beliefs are not knowledge) "posits a certain block to our judging that we know, and the changes that would clear the way to our judging that we know also remove this block." (DeRose 1995, 25). However, in case of one's belief that one is not a BIV, the connection between SCA and our judging whether we know are not that straightforward, for it is neither easy to imagine circumstances where one knows that one is not a BIV nor circumstances where this belief is sensitive. DeRose (1995, 25-26) notes:

While it's difficult to imagine a situation in which one seems to know that one's not a BIV, it's likewise difficult to imagine circumstances in which the block SCA posits is removed. It's difficult, that is, to imagine a situation in which someone believes they're not a BIV but in which the conditional If $S$ were a BIV, then $S$ would believe she wasn't a BIV isn't true.

The situation we face is that our beliefs that we are not BIVs are insensitive and we tend to judge that we do not know that we are not BIVs. In order to determine the connections between these two facts we have to consider alternative circumstances or counterfactual situations but they are not easy at hand, according to DeRose. He argues that in analogous cases a strong connection is established between the insensitivity of beliefs and our 
tendency to judge that they fail to constitute knowledge. ${ }^{3}$ DeRose claims that this connection also holds in case of our beliefs that we are not BIVs, though no alternative circumstances or counterfactual situations are easily imaginable.

In the following, I will contest this claim. I will examine the connection between the insensitivity of our beliefs that we are not BIVs and the fact that we tend to judge that these beliefs do not constitute knowledge by investigating counterfactual situations and cases of closely related anti-skeptical hypotheses. I will conclude that no such connection exists and that-maybe in contrast to our first intuition - the insensitivity of our beliefs that we are not BIVs and our tendency to judge that we do not know that we are not BIVs is a mere coincidence.

For investigating this connection, we can consider four subjunctives: ${ }^{4}$

S1 If S's belief that the skeptical hypothesis is false were sensitive, then we would judge that S knows that the skeptical hypothesis is false.

$\mathrm{S}(\sim s h) \rightarrow \mathrm{JK}(\sim s h)$

S2 If we were to judge that $S$ does not know that the skeptical hypothesis is false, then S's belief would not be sensitive.

$\mathrm{J} \sim \mathrm{K}(\sim s h) \rightarrow \sim \mathrm{S}(\sim s h)$

S3 If we were to judge that $\mathrm{S}$ knows that the skeptical hypothesis is false, then $\mathrm{S}$ 's belief would be sensitive.

$\mathrm{JK}(\sim s h) \rightarrow \mathrm{S}(\sim s h)$

S4 If S's belief that the skeptical hypothesis is false were insensitive, then we would judge that $S$ does not know that the skeptical hypothesis is false.

$\sim \mathrm{S}(\sim s h) \rightarrow \mathrm{J} \sim \mathrm{K}(\sim s h)$

The truth-conditions for these subjunctives are as follows: S1 is true iff in the nearest possible world, where S's belief that $\sim s h$ is sensitive, we judge that $\mathrm{S}$ knows that $\sim s h ; \mathrm{S} 2$ is true iff in all nearby possible worlds, where we judge that $\mathrm{S}$ does not know $\sim$ sh, this belief is insensitive; $\mathrm{S} 3$ is true iff in the nearest possible world, where we judge that S knows $\sim s h$, S's belief that $\sim s h$ is sensitive; S4 is true iff in all nearby possible worlds, where S's belief that $\sim s h$ is insensitive, we judge that $\mathrm{S}$ does not know that $\sim s h$.

In the following I will argue that none of these four subjunctives is true in a way that can establish a connection between the insensitivity of our beliefs that $\sim s h$ and our intuition that we do not know that $\sim s h$. I do not argue that these four subjunctives capture any possible connection between the insensitivity of these beliefs and our judgment that they fail to constitute knowledge, but I do not see any reason why we should assume such a connection if it is not established by any of these four subjunctives.

\footnotetext{
${ }^{3}$ Analogous cases mentioned by DeRose are "The Cubs won yesterday" and "My newspaper isn't mistaken about whether the Cubs won yesterday" or "Those animals are zebras" and "Those animals are not just cleverly painted mules".

${ }^{4}$ One might regard these subjunctives as counterfactual conditionals, but strictly speaking only S1 and S3 are counterfactuals, since the antecedents of S2 and S4 are true.
} 
Beliefs in anti-skeptical hypotheses such as the hypothesis that I am not a BIV turn out to be insensitive. However, beliefs in the conjunction of $\sim$ sh and any external world proposition $p$ turn out to be sensitive, if the belief $\mathrm{B}(p)$ itself is sensitive. The nearest possible world where a conjunction is false is one where one of its conjuncts is false (or both conjuncts). Worlds where the skeptical hypothesis is true are farther off than worlds where just $p$ is false. Hence, the nearest possible world where $\sim s h \& p$ is false is one where $p$ is false and $\sim s h$ is true. But if $\mathrm{B}(p)$ is sensitive, then $\mathrm{S}$ does not believe that $p$ in this world anymore. If $\mathrm{S}$ does not believe that $p$, then $\mathrm{S}$ also does not believe that $\sim s h \& p .{ }^{5}$ Hence, in the nearest possible world where $\sim s h \& \mathrm{p}$ is false, $\mathrm{S}$ does not believe that $\sim s h \& p$ anymore and the belief $\mathrm{B}(\sim s h \& p)$ turns out to be sensitive. Hence, it can be the case that $\mathrm{B}(\sim s h \& p)$ is sensitive but that $\mathrm{B}(\sim s h)$ is insensitive.

Nozick (1981, 227-228) already noted this peculiarity of sensitivity. He simply accepted the result that it is possible that we know $\sim s h \& p$ without knowing $\sim s h$ and that one can sometimes know a conjunction without knowing one of its conjuncts, i.e. that knowledge-closure does not hold between a conjunction and its conjuncts. Although Nozick was willing to accept this result, I assume that this is a highly counter-intuitive claim and that there is wide agreement that we do not know $\sim s h \& p$, if we do not know $\sim s h$. Moreover, I assume that DeRose finds the conjunction of knowing $\sim s h \& p$ and not knowing $\sim s h$ as abominable as those instances of closure failure that he mentions. So if there is a tendency to judge that we do not know $\sim s h$ as DeRose claims, then there is also a tendency to judge that we do not know $\sim s h \& p$. But what are our reasons for judging that we do not know that $\sim s h \& p$ ? Here, DeRose faces a dilemma. First, he can assume that there is the same reason for judging that we do not know $\sim s h \& p$ and for judging that we do not know $\sim s h$. But the reason for judging that we do not know that $\sim s h \& p$ cannot be the insensitivity of the belief, since $\mathrm{B}(\sim s h \& p)$ is sensitive. Furthermore, the reason cannot be our belief that $\mathrm{B}(\sim s h \& p)$ is insensitive, because we maintain our judgment that we do not know $\sim \operatorname{sh} \& p$, even if we realize that this belief is sensitive. But if we judge that we do not know $\sim s h$ for the same reason that we judge that we do not know $\sim s h \& p$, then this reason cannot be that the belief $\mathrm{B}(\sim s h)$ is insensitive. Briefly said, the reason why we judge that we do not know $\sim s h$ is not insensitivity because insensitivity is not the reason in case of $\sim s h \& p$.

DeRose claims that there is no possible world imaginable where S's belief that $\sim s h$ turns out to be sensitive. In this case, the counterfactuals S1 and S2 are still true, but they are vacuously true. S1 is true for the reason that its antecedent is false in all possible worlds and S2 for the reason that its consequent is true in all possible worlds. However, the case of $\mathrm{B}(\sim s h \& p)$ shows that the truth of S1 and S2 does not establish an explanatory relation between sensitivity and our judgments that we know. There are nearby possible worlds where B( sh \& $p$ ) is sensitive, namely any world where $\mathrm{B}(p)$ is sensitive. But in these possible worlds, we still judge that we do not know $\sim s h \& p$, so the instance of $\mathrm{S} 1$ for $\sim s h \& p$ is false. Furthermore, it is not the case that in all nearby possible worlds where we judge that we do not know $\sim s h \& p, \mathrm{~B}(\sim s h \& p)$ turns out to be insensitive, so the instance of $\mathrm{S} 2$ for $\sim \operatorname{sh} \& p$ is false as well. This is even false for the actual world where we judge that we do not know $\sim s h \& p$, though $\mathrm{B}(\sim s h \& p)$ is sensitive. This is the first horn of DeRose's dilemma, based on the assumption that there is the same reason for not knowing $\sim s h$ and for not knowing $\sim s h \& p$.

\footnotetext{
${ }^{5}$ This is at least the case if we consider minimally coherent persons who do not believe a conjunction, if they do not believe one of the conjuncts.
} 
One can avoid this problem by modifying the sensitivity conditions for conjunctions such that any belief $\mathrm{B}(a \&$ $b)$ is sensitive only if $\mathrm{B}(a)$ and $\mathrm{B}(b)$ were sensitive. Thus $\mathrm{B}(\sim s h \& p)$ turns out to be insensitive. In this case, insensitivity can be the reason for judging that we do not know $\sim s h$ and for judging that we do not know $\sim s h \&$ $p$. Notably, this is not a viable strategy for indirect sensitivity accounts. First, it is not in accordance with Nozick's own sensitivity account. Clearly, one can modify a sensitivity-based account of knowledge such that it better reflects our pre-theoretic intuitions about knowledge. Hence, modifying the sensitivity conditions for conjunctions is a viable strategy for direct sensitivity accounts to avoid implausible results. However, indirect sensitivity accounts such as DeRose's require sensitivity to explain our intuitions about knowledge ascriptions. But why should we accept that a modified sensitivity account explains our intuitions about knowledge ascriptions if it does not even reflect its innovator's intuitions about sensitivity? Claiming this seems ad hoc.

Moreover, by adopting this strategy, indirect sensitivity accounts would inverse the order of explanation. They aim at explaining our intuitions about knowledge ascriptions by relying on sensitivity. However, the modification to this sensitivity account would be motivated by our intuitions about knowledge, i.e. by the intuition that $\mathrm{S}$ knows each conjunct if $\mathrm{S}$ knows the conjunction. Hence, defenders of indirect sensitivity accounts would use a sensitivity account whose modification is based on our pre-theoretic understanding of knowledge for explaining our intuitions about knowledge, an explanatory circle. Therefore, modifying Nozick's sensitivity account such that beliefs of conjunctions are sensitive only if the beliefs in the conjuncts are sensitive is not a viable strategy for indirect sensitivity account, because they aim at explaining our intuitions about knowledge by relying on the notion sensitivity.

DeRose's second option is to claim that we fail to know the two propositions $\sim s h$ and $\sim s h \& p$ for different reasons, i.e. we tend to judge that we do not know $\sim s h$ because the belief $\sim s h$ is insensitive, but that we tend to judge that we do not know $\sim s h \& p$ for some other reason. First, we could assume that the reason for judging that we do not know $\sim s h \& p$ is in no way related to sensitivity at all. This assumption simply seems to deliver false results regarding our intuitions. If we assume that the reason we judge that that we do not know $\sim s h \& p$ is in no way related to insensitivity, then we should conclude that insensitivity also fails to play a crucial role in our judgments about our belief that $\sim s h$. Otherwise our judgments are totally ad hoc.

Second, we could assume that the reason for judging that we do not know $\sim \operatorname{sh} \& p$ is not insensitivity, but related to insensitivity. One could argue, for example, that we tend to judge that we do not know $\sim s h \& p$ because we came to believe $\sim s h \& p$ via inference from $\sim s h$ and $p$ and the insensitivity of beliefs of the premise $\sim s h$ explains our tendency to judge that we do not know $\sim s h \& p .{ }^{6}$ In this case, the reason for judging that we do not know $\sim s h \& p$ is not insensitivity, but still related to it. ${ }^{7}$

Moreover, we could distinguish between minimal skeptical hypotheses, such as $\sim s h$, and non-minimal skeptical hypotheses, such as $\sim s h \& p$. Based on this distinction we could argue that insensitivity is the reason for our

\footnotetext{
${ }^{6}$ I am thankful to Ernest Sosa for pointing out this option.

${ }^{7}$ One could continue to argue that $\mathrm{S}$ does not know $\sim s h \& p$ via inference from $\sim s h$ and $p$ if $\mathrm{S}$ does not know $\sim s h$ and $p$. Baumann (2012) reconstructs Nozick's remark about inferential knowledge along these lines. However, it is important to note that this account contradicts Nozick's view that $\mathrm{S}$ knows $\sim s h \& p$ but that $\mathrm{S}$ does not know $\sim s h$. I am indebted to Peter Baumann for helpful comments on this issue.
} 
tendency to judge that we do not know minimal skeptical hypotheses, but that the reason for judging that we do not know non-minimal skeptical hypotheses is not insensitivity but still related to it. ${ }^{8}$

I see two serious problems for this account. First, this explanation is not in accordance with Nozick's judgment that insensitivity is the reason for judging that we do not know $\sim s h$, but that there is no reason for judging that we do not know $\sim s h \& p$, because the belief in the conjunction can be sensitive and, therefore, is able to constitute knowledge. Hence, there would not be agreement about knowledge judgments concerning $\sim s h \& p$ within the community of defenders of sensitivity accounts. However, given this disagreement even within this community, I do not think that this account can explain our skeptical intuitions in general, as DeRose's indirect sensitivity account demands.

Second, this account can only apply if $\mathrm{S}$ comes to believe $\sim s h \& p$ via inference from $\sim s h$ and $p$. However, if the belief-forming process is a different one, we still tend to judge that $\mathrm{S}$ does not know $\sim s h \& p$, but this fact can no longer be explained by the suggested account.

To sum up, DeRose faces the following dilemma: He either has to confess that the connections that S1 and S2 suggest do not hold, because S1 and S2 are only vacuously true or he has to make the problematic claim that we tend to judge that we do not know $\sim s h$ for a different reason than we tend to judge that we do not know $\sim s h \& p$. Moreover, I do not see any successful strategy for solving this problem in one way or the other.

DeRose is in no better position with respect to the subjunctives S3 and S4. S3 is true iff in the nearest possible world, where we judge that S knows $\sim s h$, this belief is sensitive; S4 is true iff in all nearby possible worlds, where S's belief that $\sim s h$ is insensitive, we judge that $\mathrm{S}$ does not know that $\sim s h$. Both subjunctives turn out to be false if there are nearby possible worlds where we judge that $\mathrm{S}$ knows $\sim s h$ but $\mathrm{S}$ 's belief that $\sim s h$ is insensitive. S3 and S4 turn out to be false, if we take inferences to the best explanation as potential solutions to the skeptical problem into account. Inferences to the best explanation are usually regarded as a potential knowledge source, although the resulting beliefs are insensitive. Here is an example: I see that the street is wet and I come to know via inference to the best explanation that it has been raining. However, the resulting belief is insensitive, for I would still come to believe that it has been raining via inference to the best explanation from my belief that the street is wet if it had not been raining and something else had caused the street being wet.

Inferences to the best explanation are one anti-skeptical strategy among others. ${ }^{9}$ Representatives of this account argue that the existence of an external world which is broadly the way we perceive it to be offers the best explanation for our experiences and not something else like in BIV-scenarios. They conclude that we can have external world knowledge and knowledge that the skeptical hypothesis is false via inference to the best explanation. Such beliefs of $\sim s h$ formed via inferences to the best explanation turn out to be insensitive for we would still believe $\sim s h$ via inference to the best explanation if we were BIVs. However, if it is possible that $\mathrm{S}$ knows via inference to the best explanation that $\sim s h$, then S3 and S4 turn out to be false. S3 is false because there is a (close) possible world where we tend to judge that $\mathrm{S}$ knows $\sim s h$, but this belief is still insensitive. ${ }^{10} \mathrm{~S} 4$

\footnotetext{
${ }^{8}$ I am thankful to an anonymous reviewer of Erkenntnis for this suggestion.

${ }^{9}$ See for example Vogel (1990) for such an account.

${ }^{10} \mathrm{We}$ could also assume, if need so, that this possible world is the nearest possible world by assuming that inferences to the best explanation are the only possible way to come to know $\sim s h$.
} 
is false because there are nearby possible worlds where S's belief is still insensitive, but we tend to judge that $S$ knows that $\sim s h$. Hence, if knowledge that $\sim s h$ via inference to the best explanation is possible, then S3 and S4 turn out to be false.

It is controversial whether such an inference to the best explanation that $\sim s h$ is true has already been found and most philosophers probably even doubt that it can ever be found. However, this is not the crucial point. The point is that we do not doubt that we know that $\sim s h$ via inference to the best explanation for the reason that the resulting belief would be insensitive, but for the reason that those explanations for $\sim s h$ that are actually available and those that are possibly available are really the best ones. Hence, the fact that we do not abandon knowledge via inference to the best explanation for the reason that the resulting belief is insensitive already offers counterevidence against the view that we judge S3 and S4 to be true.

To sum up: Our beliefs that the skeptical hypothesis is false are insensitive and there is a tendency to judge that we do not know that the skeptical hypothesis is false. There might seem to be a connection between these two facts at first sight, but a careful investigation reveals that such a connection does not exist. The insensitivity of our beliefs that $\sim s h$ and our tendency to judge that we do not know $\sim s h$ are just coincidental. ${ }^{11}$

\section{Conclusion}

DeRose suggests that by asserting that one knows (or that one does not know) $\sim s h$ we raise the standards, if need so, up to a level where sensitivity is required for knowledge. Assertions that we do know $\sim s h$ turn out to be false when raising the standards, and assertions that we do not know $\sim s h$ turn out to be true. DeRose can still claim that by making such assertions we raise the standards up to a level where sensitivity is required, but this claim is totally ad hoc given the fact that he cannot establish any connection between the insensitivity of our beliefs that $\sim s h$ and the tendency to judge that we do not know $\sim s h$. Hence, DeRose's indirect sensitivityaccount is simply not motivated. DeRose is mistaken that his sensitivity account is "on the right track". However, DeRose can abandon his sensitivity-account and still defend a contextualist solution to the skeptical problem, but in this case his version of contextualism does not incorporate an explanation why we raise the standards anymore which DeRose regards as the central advantage of his account over alternative contextualist accounts.

${ }^{11}$ DeRose's account faces an additional problem that I will not handle here in detail. He claims that we only raise the standards by asserting full-fledged skeptical hypotheses (or their denials) which not only contain the claim that we falsely believe but also an explanation why we falsely believe. Thus by just asserting that our beliefs are false we do not raise the standards. Here, DeRose draws a distinction between two kinds of skeptical hypotheses in order to handle our intuition that we do not know that we are BIVs deceived in falsely believing while we do know that our beliefs are not false. However, DeRose is mistaken. Moore's (1939) formulated his proof of an external world in a way that it is not a reaction to a skeptical hypothesis containing an explanation why we are deceived; still we regard this 'proof' not as less problematic than a 'Moorean' argument objecting to a full-fledged skeptical hypothesis. Hence, some skeptical hypotheses' missing explanation why we falsely believe, does not explain, why we do not raise the standards by asserting them, because sometimes we do raise the standards in such cases. 


\section{Acknowledgements}

The research was funded by the Austrian Science Fund (FWF): J 3174-G15. I am indebted to Ernest Sosa and the members of his epistemology group at Rutgers University for their helpful comments, to Martina Fürst, Lisa Miracchi and Danilo Šuster for their remarks on earlier drafts of this paper and to Peter Klein for insightful and encouraging discussions.

\section{References}

Adams, F. and Clarke, M. (2005). Resurrecting the tracking theories. Australasian Journal of Philosophy, 83(2), 207-221.

Baumann, P. (2012). Nozick's defense of closure. (In K. Becker and T. Black (Eds.), The sensitivity principle in epistemology (pp. 11-27). Cambridge: Cambridge University Press.)

David, M. and Warfield, T. (2008). Knowledge-closure and skepticism. (In Q. Smith (Ed.), Epistemology: New essays (pp. 137-187), Oxford: Oxford University Press.)

DeRose, K. (1995). Solving the skeptical problem. The Philosophical Review, 104(1), 1-52.

DeRose, K. 2010. Insensitivity is back, baby!. Philosophical Perspectives, 24, 161-187.

Kripke S. A. (2011). Nozick on knowledge. (In Philosophical troubles. Collected papers, volume I (pp. 162224). Oxford: Oxford University Press.)

Moore, G. E. (1939). Proof of an external world. Proceedings of the British Academy, 25, 273-300.

Nozick, R. (1981). Philosophical explanations, (Cambridge: Cambridge University Press.)

Sosa, E. (1999). How to defeat opposition to Moore. Philosophical Perspectives, 13, 141-153.

Vogel, J. (1987). Tracking, closure and inductive knowledge. (In S. Luper-Foy (Ed.), The possibility of knowledge: Nozick and his critics, (pp. 197-215). Totowa, NJ: Rowman and Littlefield.)

Vogel, J. (1990). Cartesian skepticism and inference to the best explanation. Journal of Philosophy 87(11), 658666.

Williamson, T. (2000). Knowledge and its limits, (Oxford: Oxford University Press.) 